\title{
Le fonds Proust au département des Manuscrits de la Bibliothèque nationale de France
}

Notes pour un cinquantenaire

\section{Guillaume Fau}

\section{OpenEdition}

\section{Journals}

Édition électronique

URL : http://journals.openedition.org/genesis/1154

DOI : 10.4000/genesis. 1154

ISSN : 2268-1590

Éditeur :

Presses universitaires de Paris Sorbonne (PUPS), Société internationale de génétique artistique littéraire et scientifique (SIGALES)

\section{Édition imprimée}

Date de publication : 15 juin 2013

Pagination : 135-140

ISBN : 978-2-84050-893-9

ISSN : $1167-5101$

Référence électronique

Guillaume Fau, «Le fonds Proust au département des Manuscrits de la Bibliothèque nationale de France », Genesis [En ligne], 36 | 2013, mis en ligne le 08 juillet 2015, consulté le 20 avril 2019. URL http://journals.openedition.org/genesis/1154; DOI : 10.4000/genesis.1154 


\section{Le fonds Proust au département des Manuscrits de la Bibliothèque nationale de France Notes pour un cinquantenaire}

Guillaume Fau

$\mathrm{S}$ i le temps de la création est du domaine de la génétique des textes - comment, en une quinzaine d'années seulement, de 1908 environ à sa mort en 1922, Marcel Proust a-t-il conçu, écrit, remanié, corrigé et, pour partie, publié le texte d'À la recherche du temps perdu, et selon quelles étapes ? -, le temps de la conservation publique des archives - cinquante ans en 2012 depuis l'arrivée du fonds à la Bibliothèque nationale en 1962 relève du domaine plus prosaïque de la bibliothéconomie : temps de l'institution et des opérations de traitement qu'elle met en œuvre. Retracer l'histoire de la conservation du fonds Proust, c'est sauver de l'oubli ces données administratives et techniques qui ont contribué, depuis un demi-siècle, à façonner l'aspect des manuscrits tels qu'ils sont aujourd'hui accessibles aux chercheurs.

\section{Trois acquisitions majeures et leur signalement}

En 1962, la Bibliothèque nationale, sous l'administration de Julien Cain, achète à la nièce de Proust, Suzy Mante-Proust, les manuscrits restés en la possession de la famille du frère de l'écrivain, Robert Proust. Avec cette acquisition'1, entrent dans les collections nationales les manuscrits d'À la recherche du temps perdu comme ceux des œuvres de jeunesse. Par la suite, deux autres acquisitions importantes sont venues compléter le fonds : un reliquat de fragments, «paperoles » et correspondances diverses, acheté à Suzy Mante-Proust en $1977^{2}$, et treize cahiers de brouillon de la Recherche, issus de la collection de Jacques Guérin, entrés au département des Manuscrits en $1983^{3}$.

Faut-il rappeler que, jusqu'en 2000, Florence Callu fut le maître d'œuvre du colossal chantier d'accroissement, de classement, de signalisation, de restauration, de sauvegarde et de valorisation du fonds, fleuron des collections modernes et contemporaines du département des Manuscrits 4 ?

L'essentiel des manuscrits de Marcel Proust conservés aujourd'hui à la BnF dans le fonds des Nouvelles acquisitions françaises (NAF), s'organise donc de la façon suivante :

- NAF 16611-16781. Fonds Marcel Proust. Cet ensemble, qui constitue le cœur de la collection, est issu de l'achat de 1962. L'inventaire du fonds répertorie cent soixante et onze numéros, décrits selon le plan de classement suivant : I. Euvres de jeunesse (I, Papiers scolaires ; II-IV, Les Plaisirs et les jours ; V-VI, Jean Santeuil ; VII-XXI, Traductions de Ruskin ; XXII-XXIII, Pastiches et mélanges; XXIV-XXV, Chroniques ; XXVI, Contre Sainte-Beuve); II. À la recherche du temps perdu (XXVII-CXIX, Manuscrits autographes ; CXX-CXLII, Dactylographies ; CXLIII-CLXVI, Épreuves ; CLXVIICLXXI, Bonnes feuilles et éditions).

- NAF 18313-18325. Cahiers de brouillon contenant des ébauches des différentes parties de la Recherche, à divers stades de leur rédaction. Il s'agit des treize cahiers entrés en 1983, en complément des soixante-deux arrivés en 1962. Deux modes d'acquisition cumulés ont permis cet accroissement ${ }^{5}$ : dation en paiement des droits de succession pour les neuf cahiers cotés NAF 18314-18317,

\footnotetext{
1. Achat, 1962. A. 23894.

2. Achat, 1977. A. 26803.

3. Achat et dation en paiement des droits de succession, 1983. A. 83-10.

4. Florence Callu, « Le fonds Proust de la Bibliothèque nationale », RTP,

I, p. CXLV-CXLVII.

5. Florence Callu, « Le régime des dations à la Bibliothèque nationale : 1'acquisition de manuscrits de Marcel Proust », Études sur la Bibliothèque nationale et témoignages réunis en hommage à Thérèse Kleindienst, Paris, Bibliothèque nationale, 1985, p. 205-209.
} 
18320-18323, 18325, que Jacques Guérin avait achetés à Madame Robert Proust en 1936 par l'entremise du libraire Lefèvre ; achat des quatre cahiers cotés NAF 18313, $18318,18319,18324$ (les trois premiers acquis par Jacques Guérin en 1962, le dernier acheté en 1936 à Madame Robert Proust par l'entremise du libraire Lefèvre).

- NAF 27350-27352. Fragments d'œuvres et correspondance. Cet ensemble de documents disparates (feuillets de cahiers, paperoles, dactylographies, lettres) provient du « cartonnier » dont le contenu fut acheté en 1977.

L'inventaire des deux premières tranches de cotes a été publié dans le dernier volume imprimé du catalogue des Nouvelles acquisitions françaises paru en 19996. Au tournant des années 2000, la Bibliothèque nationale de France a fait le choix de la $\mathrm{DTD} \mathrm{EAD}^{7}$ pour décrire les manuscrits conservés au département des Manuscrits, au département des Arts du spectacle et à la Bibliothèque de l'Arsenal. Depuis lors, les notices du fonds Proust, issues de la conversion rétrospective du catalogue imprimé et du catalogage courant, sont accessibles en ligne à l'adresse du catalogue BNF archives et manuscrits (<http://archivesetmanuscrits. bnf.fr/>). L'encodage des données d'inventaire en EAD a permis, notamment, de créer un lien entre la notice et l'image numérisée du manuscrit dans Gallica.

\section{Restauration}

À son arrivée en 1962, le fonds Proust fut l'objet d'une vaste campagne de restauration qui, menée de front avec le travail de classement, s'étendit jusqu'à la fin de la décennie. Trois grands types d'interventions furent mis en œuvre, selon la nature des documents. Les cahiers posèrent les problèmes les plus nombreux : papiers abîmés, feuillets cassants, bords fragilisés, coutures menaçant de lâcher, paperoles déchirées, décollées ou égarées... Leur restauration systématique mobilisa Florence Callu et les restaurateurs de la Bibliothèque nationale pendant de longs mois. Les pièces en feuilles (dactylographies et épreuves, pour l'essentiel), quant à elles, furent reliées en volumes, méthode de conservation alors systématiquement adoptée par le département des Manuscrits de la Bibliothèque nationale. Enfin, à ces mesures s'ajoutèrent celles requises, d'une manière plus dramatique encore car il y allait de la survie matérielle des documents et de leur communicabilité aux chercheurs, par les vingt cahiers de mise au net du texte de la Recherche (NAF 16708-16727) : " C'est dans ces cahiers que se trouvent les innombrables béquets ou paperoles que Marcel Proust s'est plu à ajouter sans relâche à son œuvre. Le texte est écrit généralement sur le recto de chaque feuille de papier à laquelle sont ajoutées en haut et en bas plusieurs autres feuilles, collées bout à bout en longues bandes qui peuvent atteindre dans les cas extrêmes un mètre quarante. Les problèmes posés par la conservation de ces bandes sont très difficiles à résoudre et il faut envisager le plus souvent de les doubler d'une mousseline de soie pour les préserver de l'effritement » (note de Florence Callu, contemporaine de la restauration du fonds). En fait, l'état de conservation de ces manuscrits, d'un type inédit et pour lesquels il fallut inventer des solutions sur mesure, requit, outre le doublage du papier, une restauration du cahier dans son ensemble du fait même de la surabondance des paperoles. Les quelques images reproduites dans l'ouvrage de Pierre Abraham, Proust, recherches sur la création intellectuelle (Paris, Rieder, 1930), ne donnent qu'une petite idée de l'état des cahiers et de leurs couvertures avant restauration (fig. 1), mais le débordement des paperoles à l'extérieur du format du cahier y est bien apparent. Par mesure de sauvegarde, il fut donc décidé de procéder au démontage de chacun de ces vingt cahiers puis à leur reliure sur onglets piqués, le bloc du cahier et les paperoles se trouvant ainsi protégés et maintenus. L'ouverture des volumes s'en est trouvée également facilitée au moment de leur numérisation, bien des années plus tard (fig. 2 et 3 ).

Toutes ces mesures de restauration et de préservation furent effectuées selon les préconisations de l'époque, dans le respect du principe de réversibilité, par l'atelier de restauration de la Bibliothèque nationale. Elles se sont achevées avec la mise en boîtes de conservation, confectionnées sur mesure de 1974 à 1975. En 1987, des boîtes similaires furent réalisées pour les treize cahiers

6. Catalogue des Nouvelles acquisitions françaises du département des Manuscrits, 1972-1986, nos 16428-18755, Paris, Bibliothèque nationale de France, 1999, p. 20-34 et 174-175.

7. Voir <www.bnf.fr/fr/professionnels/formats_catalogage/a.f_ead. html>. 


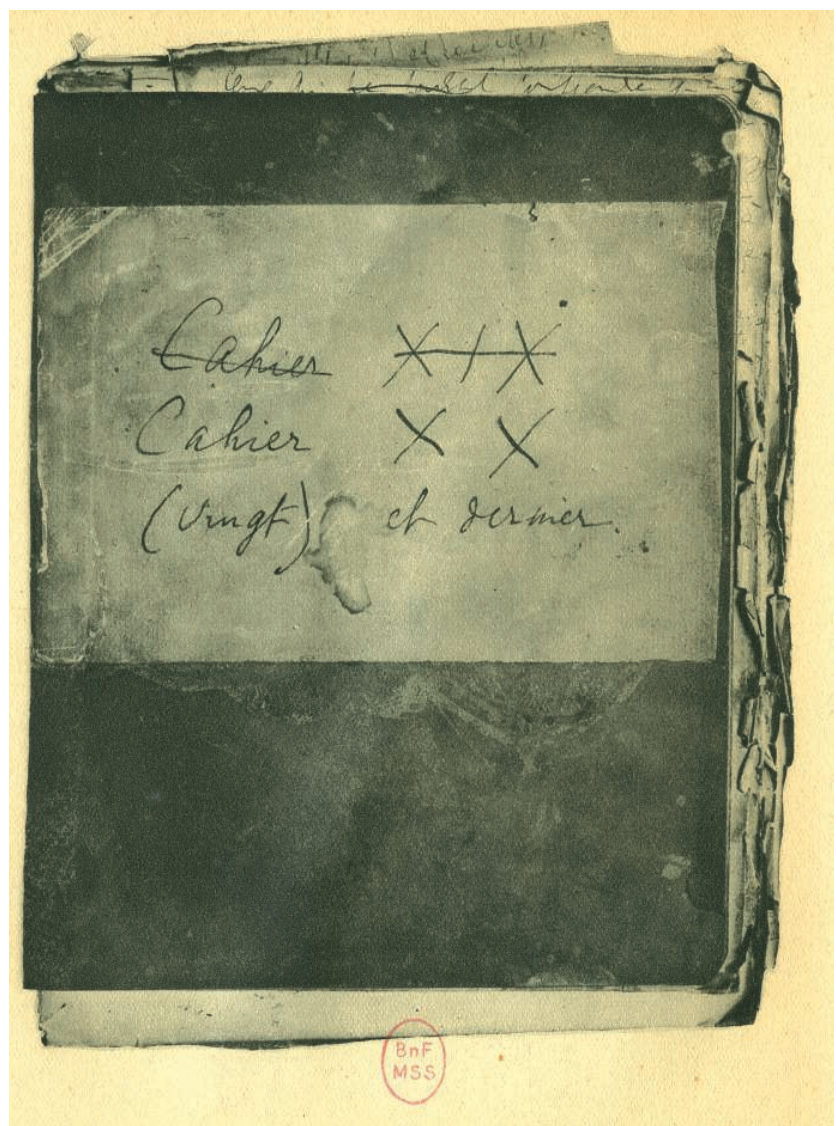

Fig. 1 : Le Cahier XX (NAF 16727) avant restauration. Illustration tirée de l'ouvrage de P. Abraham, Proust, recherches sur la création intellectuelle (Paris, Rieder, 1930, p. LX)

arrivés en 1983. Enfin, les éléments du « cartonnier », acquis en 1977 et qui avaient exigé un long travail d'identification, furent reliés en quatre volumes, de 2004 à 2007. En tête du volume coté NAF 27350 (1), une table établit la concordance entre les fragments retrouvés et les cahiers conservés dans le fonds auxquels ils se rapportent.

\section{Des microfilms à la numérisation}

La constitution d'une collection de microfilms de substitution fut décidée dès l'arrivée du fonds en 1962. Effectué au fur et à mesure de l'avancement du
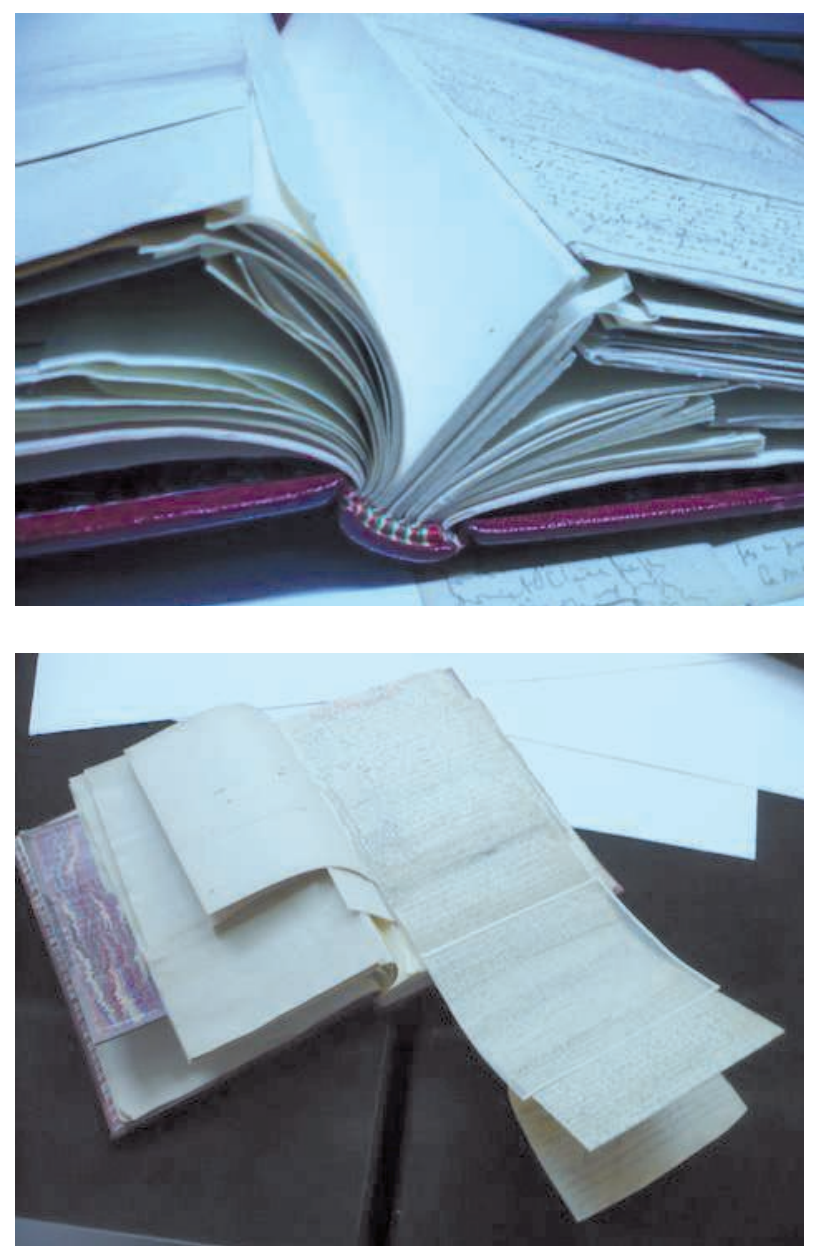

Fig. 2 et 3 : Les cahiers de la mise au net restaurés et montés sur onglets piqués

classement et de la restauration, le microfilmage répondit aussi, tout au long des années soixante, aux nombreuses et pressantes commandes de reproduction portant sur des pans entiers de la collection, émanant essentiellement, dès 1963, de grandes bibliothèques américaines (Houghton Library, à Harvard, bibliothèques des universités du Michigan, d'Illinois...). Le tarissement des commandes américaines de reproduction de masse, aux alentours de 1969, pourrait laisser penser que la couverture microfilm du fonds est quasi exhaustive à cette date. Les acquisitions ultérieures, quant à elles, furent microfilmées de mai 1984 à mai 1985 (achat et dation de 1983). 
Les vingt cahiers de la mise au net du texte de la Recherche constituent un cas particulier. Ils semblent avoir été microfilmés une première fois par la Bibliothèque nationale dès 1947 , pour le compte de Suzy Mante-Proust qui en était alors encore propriétaire. La consultation de ces microfilms, utilisés par Pierre Clarac et André Ferré pour l'édition de la «Bibliothèque de la Pléiade » de $1954^{8}$, permettrait aujourd'hui de mieux comprendre l'état de ces vingt cahiers avant leur restauration à la Bibliothèque nationale dans les années soixante.

En 2008, le département des Manuscrits a fait le choix d'abandonner le microfilmage de ses collections à des fins de sauvegarde au profit de la numérisation. De 2003 à 2011, les programmes de numérisation du fonds Proust ont constitué un temps fort de cette (r)évolution des procédures de conservation mais aussi de communication et de valorisation des fonds patrimoniaux. Le pilotage de ces projets fut assuré en partenariat par le département des Manuscrits de la BnF et l'équipe Proust de l'ITEM, dirigée par Nathalie Mauriac.

Un premier projet, en guise de galop d'essai, fut proposé par l'ITEM en 2003 à Monique Cohen, alors directrice du département des Manuscrits. Intitulé IDA, il a permis, dans le cadre du programme "Société de l'information » du CNRS dirigé par Jean-Louis Lebrave, de numériser un premier cahier de brouillon (NAF 16694 [Cahier 54]), contenant des brouillons de Sodome et Gomorrhe II et d'" Albertine disparue ». Réalisée intramuros, cette numérisation a permis aux services de la $\mathrm{BnF}$ et à l'ITEM d'élaborer une méthodologie de travail concertée dont ont bénéficié les projets ultérieurs : numérisation intégrale en TIFF couleurs $400 \mathrm{dpi}$, feuillets vierges compris, de la double page du cahier afin de donner l'image fidèle du parcours de l'écriture proustienne ; mise au point du protocole de numérisation des paperoles (ordre des prises de vues; vues d'ensemble paperoles fermées puis ouvertes ; vues de détail). La préparation du cahier pour la numérisation a aussi été l'occasion d'opérations de restauration (repositionnement au folio 34 d'une paperole rattachée par erreur au folio 35) et d'enrichissement des données descriptives (voir la notice NAF 16694 détaillée, consultable en ligne dans BNF archives et manuscrits). C'est alors également qu'une minuscule paperole (5 $\mathrm{mm}$ pliée), longtemps passée inaperçue, fut découverte au bord inférieur du folio 80 (« Nota Bene sur la marge »).

Les deux programmes de numérisation qui ont suivi ont répondu à des appels à projets de l'Agence nationale de la recherche (ANR). Pilotés en partenariat par le département des Manuscrits, sous la direction de Thierry Delcourt, et par l'ITEM, sous celle de Pierre-Marc de Biasi puis de Nathalie Mauriac, ils ont constitué, tant par leur volumétrie que par le prestige attaché aux corpus retenus, une montée en puissance de la numérisation des fonds littéraires modernes et contemporains.

De 2007 à 2010, le projet Optima («Outils Pour le Traitement et l'analyse de l'Information dans les MAnuscrits modernes ») a porté sur trois corpus de manuscrits choisis pour leurs spécificités génétiques (écriture de type scénarique de Gustave Flaubert, thématique de Paul Valéry et par unité textuelle proliférante dans le cas de Marcel Proust). À la BnF, une procédure de marché public a retenu l'offre de la société Jouve pour la numérisation. Le corpus proustien retenu était constitué des soixante-quinze cahiers de brouillon de Contre Sainte-Beuve et de la Recherche du temps perdu. Les quelque 6530 images numériques de ces cahiers (facturées 15750 euros TTC) ont été réalisées du 4 septembre 2007 au 22 octobre 2008 dans les locaux du prestataire, à Mayenne.

De 2009 à 2011, le projet Cahiers-Proust a réuni en partenariat la $\mathrm{BnF}$ (département des Manuscrits, département de la Conservation - services Numérisation et Restauration), l'ITEM (équipe Proust) et le département de littérature française de l'université de Kyoto représenté par Kazuyoshi Yoshikawa. Il a porté sur les vingt cahiers dits de «mise au net » de Sodome et Gomorrhe au Temps retrouvé (NAF 1670816727), sur les trois cahiers de mise au net du Côté de Guermantes II (NAF 16705-16707), sur les quatre carnets (NAF 16637-16640) et sur quelques reliquats (NAF 16703, NAF 16729, NAF 27350 -1 et 2). En raison de la complexité et de la fragilité extrêmes de certains des documents à numériser (cahiers démembrés

8. André Ferré, « Inédits d'Albertine disparue », BSAMP, no 2, 19511952, p. 17-18. 
et entièrement remontés sur onglets comportant des paperoles proliférantes, de taille très variable), il a été décidé de procéder à la numérisation in situ afin de mieux pouvoir encadrer les opérations de prise de vue. C'est donc à l'atelier de numérisation du service Restauration du site Richelieu de la BnF, sous la direction de Jean-Yves Sarazin puis d'Isabelle Rollet, que se sont déroulées les opérations de numérisation des quelque 6500 images, du 1er avril 2009 au 31 décembre 2011 (voir fig. 4 à 6). La subvention de l'ANR pour ce projet fut de 17000 euros TTC.

À ce jour, l'accès libre et gratuit à l'intégralité de ces images s'effectue en ligne aux trois adresses suivantes : $<$ http://archivesetmanuscrits.bnf.fr/>, pour une recherche via le catalogue illustré des collections de manuscrits de la $\mathrm{BnF}$; < http://gallica.bnf.fr/>, pour une recherche via la bibliothèque numérique de la $\mathrm{BnF}$; <www.item.ens. fr/index.php?id=578147>, pour un accès centralisé via le site de l'ITEM.

Les fac-similés papier de quatre premiers cahiers (NAF 16694, NAF 18321, NAF 16666, NAF 16693) sont également disponibles dans le cadre de la coédition $\mathrm{BnF} /$ Brepols Publishers en cours, dirigée par Nathalie Mauriac depuis 2008, dont le comité éditorial comprend en outre Bernard Brun (ITEM), Antoine Compagnon (Collège de France), Pierre-Louis Rey (Université de la Sorbonne nouvelle-Paris III) et Kazuyoshi Yoshikawa (Université de Kyoto). Florence Callu, Jean Milly, Michel Raimond et Jean-Yves Tadié en constituent le comité d'honneur.

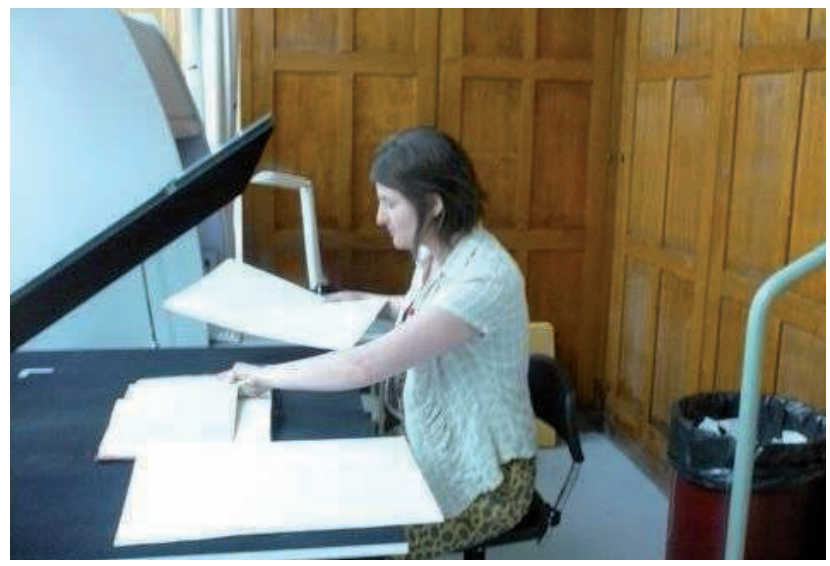

Fig. 4 : Préparation des cahiers pour la numérisation : insertion des caches

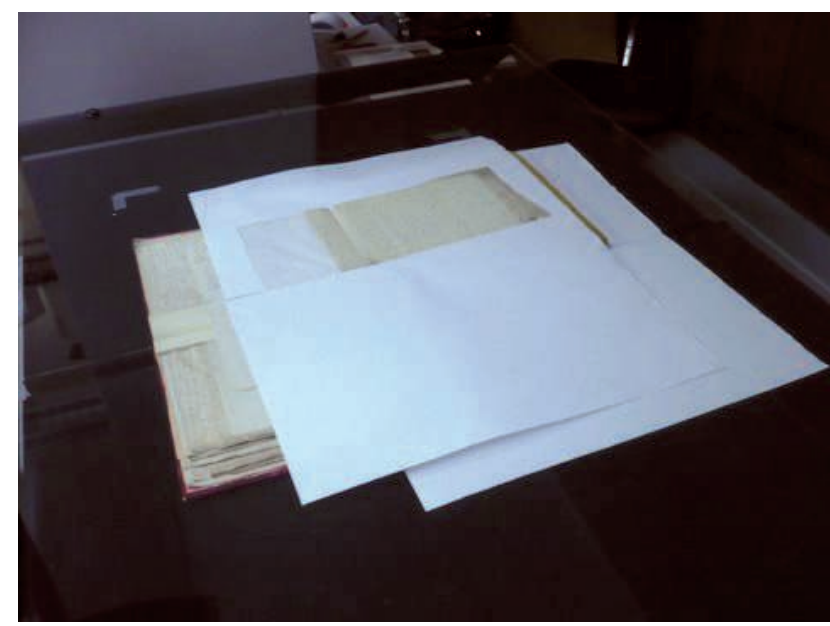

Fig. 5 : Mise à plat du feuillet à numériser

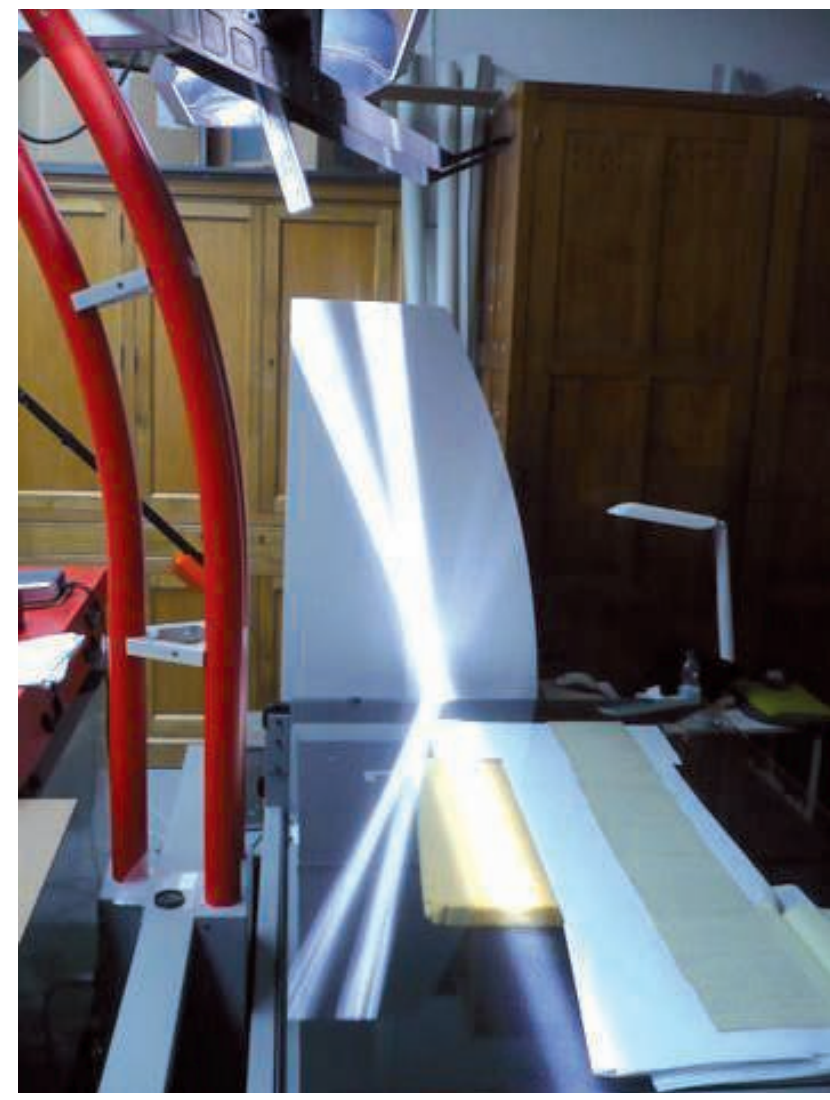

Fig. 6 : Numérisation d'une paperole 
En complément des manuscrits dont il vient d'être question, la numérisation des cinquante-quatre volumes de dactylographies et placards corrigés et des vingt-six volumes d'œuvres diverses représente désormais l'avenir à court terme de cette très riche histoire de la conservation du fonds. D'ores et déjà, les placards corrigés de $D u$ côté de chez Swann (NAF 16753), numérisés à l'occasion de l'exposition du centenaire à la Morgan Library, à New York (15 février-28 avril 2013), sont en ligne dans Gallica.

Guillaume Fau, conservateur de bibliothèque, est chef du service des manuscrits modernes et contemporains au département des Manuscrits de la Bibliothèque nationale de France. Il a organisé les expositions « Antonin Artaud » (2006), « Gaston Leroux, de Rouletabille à Chéri-Bibi » (2008) et prépare une exposition consacrée à Pierre Jean Jouve. 